\title{
The ALICE detector and trigger strategy for diffractive and electromagnetic processes
}

\author{
R. Schicker, ${ }^{a *}$ on behalf of the ALICE Collaboration \\ a Physikalisches Institut, Philosophenweg 12, 69120 Heidelberg
}

The ALICE detector at the Large Hadron Collider (LHC) consists of a central barrel, a muon spectrometer, zero degree calorimeters and additional detectors which are used for trigger purposes and for event classification. The main detector systems of relevance for measuring diffractive and electromagnetic processes are described. The trigger strategy for such measurements is outlined. The physics potential of studying diffractive and electromagnetic processes at the LHC is presented by discussing possible signatures of the Odderon.

\section{The ALICE Experiment}

The ALICE experiment is presently being built and commissioned at the Large Hadron Collider (LHC) 12 2. The ALICE experiment consists of a central barrel covering the pseudorapidity range $-0.9<\eta<0.9$ and a muon spectrometer in the range $-4.0<\eta<-2.4$. Additional detectors for trigger purposes and for event classification exist such that the range $-4.0<\eta<5.0$ is covered. The ALICE physics program foresees data taking in $\mathrm{pp}$ and $\mathrm{PbPb}$ collisions at luminosities up to $\mathcal{L}=5 \times 10^{30} \mathrm{~cm}^{-2} \mathrm{~s}^{-1}$ and $\mathcal{L}=10^{27} \mathrm{~cm}^{-2} \mathrm{~s}^{-1}$, respectively. An asymmetric system $\mathrm{pPb}$ will be measured at a luminosity of $\mathcal{L}=10^{29} \mathrm{~cm}^{-2} \mathrm{~s}^{-1}$.

\section{The ALICE Central Barrel}

The detectors in the ALICE central barrel track and identify hadrons, electrons and photons in the pseudorapidity range $-0.9<\eta<0.9$. The magnetic field strength of $0.5 \mathrm{~T}$ allows the measurement of tracks from very low transverse momenta of about $100 \mathrm{MeV} / \mathrm{c}$ to fairly high values of about $100 \mathrm{GeV} / \mathrm{c}$. The tracking detectors are designed to reconstruct secondary vertices resulting from decays of hyperons, D and $\mathrm{B}$ mesons. The granularity of the central barrel detectors is chosen such that particle tracking and identification can be achieved in a high mul-

\footnotetext{
*This work is supported in part by German BMBF under project 06HD197D
}

tiplicity environment of up to 8000 particles per unit of rapidity. The main detector systems for these tasks are the Inner Tracking System, the Time Projection Chamber, the Transition Radiation Detector and the Time of Flight array. These systems cover the full azimuthal angle within the pseudorapidity range $-0.9<\eta<0.9$ and are described below. Additional detectors with partial coverage of the central barrel are a PHOton Spectrometer (PHOS), an electromagnetic calorimeter (EMCAL) and a High-Momentum Particle Identification Detector (HMPID).

\subsection{The Inner Tracking System}

The Inner Tracking System (ITS) consists of six cylindrical layers of silicon detectors at radii from $4 \mathrm{~cm}$ to $44 \mathrm{~cm}$. The minimum radius is determined by the beam pipe dimensions whereas the maximum radius chosen is determined by the necessity of efficient track matching with the outer detectors in the central barrel. The innermost layer extends over the range $-2<\eta<2$ such that there is continous overlap with event classification detectors outside of the central barrel. Due to the high particle density of up to 80 particles $/ \mathrm{cm}^{2}$ and in order to achieve the required tracking resolution, pixel detectors have been chosen for the first two layers. Silicon drift detectors are located in the middle two layers whereas double sided silicon strip detectors are in the outer two layers. 


\subsection{The Time Projection Chamber}

The Time Projection Chamber (TPC) is the main tracking detector in the central barrel. The inner and outer radii of the active volume are 84.5 $\mathrm{cm}$ and $246.6 \mathrm{~cm}$, respectively. The full radial track length is measured in the pseudorapidity range $-0.9<\eta<0.9$ whereas tracks with at least one third of nominal radial length are covered in the pseudorapidity range $-1.5<\eta<1.5$. Particle identification is achieved by measuring the specific ionization loss. The chosen geometry results in a drift time of about $90 \mu \mathrm{s}$. This long drift time is the factor limiting the proton-proton luminosity to the value mentioned above.

\subsection{The Transition Radiation Detector}

The principal goal of the Transition Radiation Detector (TRD) is to provide electron identification in the momentum range larger than $1 \mathrm{GeV} / \mathrm{c}$. In this range, the electron identification by energy loss in the TPC is no longer sufficient. Since the TRD is a fast tracker, the TRD information can be used for an efficient trigger on high transverse momentum electrons. In addition, the position information from the TRD system improves the tracking performance of the central barrel.

\subsection{The Time of Flight Detector}

The Time-Of-Flight (TOF) array is located at a radial distance from $3.7 \mathrm{~m}$ to $4.0 \mathrm{~m}$. The TOF information is used for particle identification in the range $0.2 \mathrm{GeV} / \mathrm{c}<p_{T}<2.5 \mathrm{GeV} / \mathrm{c}$. For this detector, the Multi-gap Resistive-Plate (MRPC) technology was chosen. A strip with an active area of $120 \times 7.4 \mathrm{~cm}^{2}$ consists of pads of $3.5 \mathrm{~cm}$ length and $2.5 \mathrm{~cm}$ width.

\subsection{The Central Barrel Performance 2.5.1. Tracking Resolution}

The ITS, TPC and TRD detectors described above are the main tracking detectors in the central barrel. With the information from these detectors, particles with momenta as low as 100 $\mathrm{MeV} / \mathrm{c}$ can be tracked.

Fig 1 shows the transverse momentum resolution as expected from simulations. The TPC alone achieves a resolution of approximately $3 \%$ at a transverse momentum of $p_{T}=10 \mathrm{GeV} / \mathrm{c}$. Adding the information from ITS and TRD on

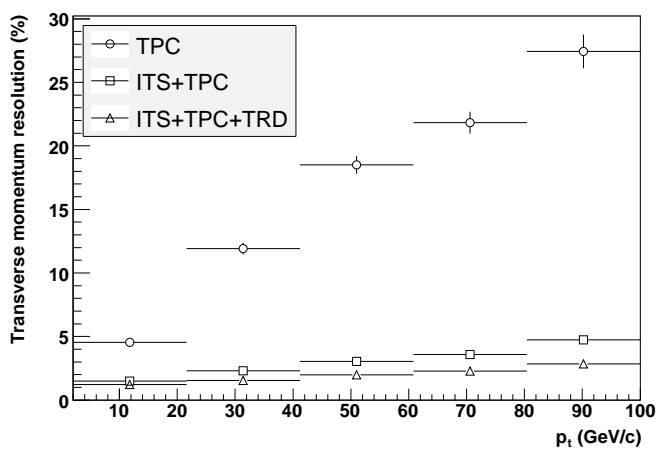

Figure 1. Central barrel tracking resolution

the inner and outer side, respectively, improves the resolution considerably due to the increased leverage. The combined transverse momentum resolution from the ITS, TPC and TRD detector is expected to be about $3 \%$ at a transverse momentum of $p_{T}=100 \mathrm{GeV} / \mathrm{c}$.

\subsubsection{Particle identification}

Particle identification is achieved in the central barrel by different methods. First, the specific energy loss is measured by the TPC, the TRD and the strip and drift detectors of the ITS.

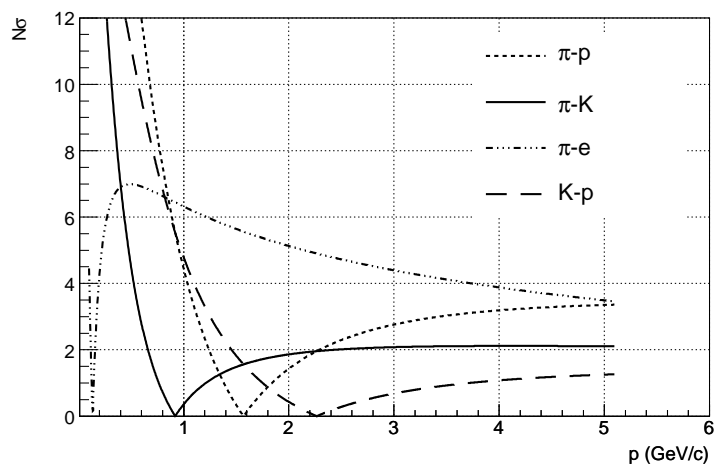

Figure 2. Particle identification by $\mathrm{dE} / \mathrm{dx}$ measurement 
Fig 2 shows the combined particle identification capability by $\mathrm{dE} / \mathrm{dx}$ measurement as a function of momentum. The separation of different particle species is shown in units of the resolution of the $\mathrm{dE} / \mathrm{dx}$ measurement.

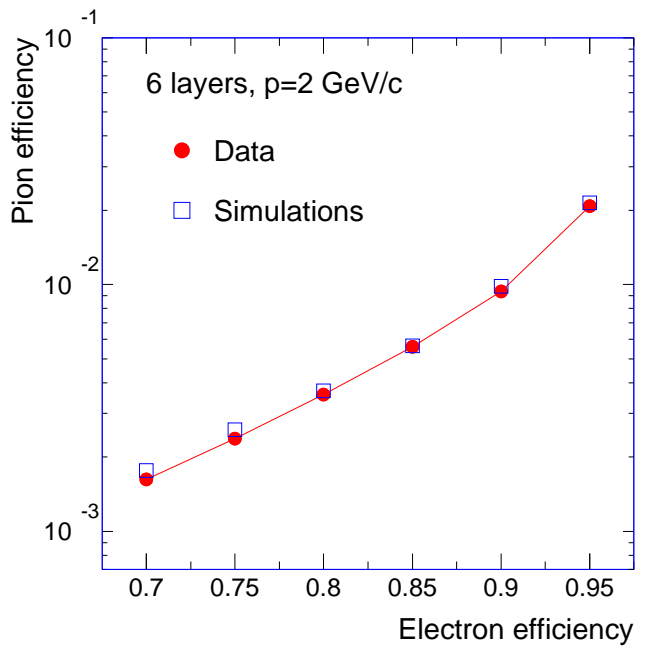

Figure 3. Electron-pion separation in the TRD

The electron-pion separation at high momenta is significantly improved by the information of the TRD system. Fig 3 shows the pion efficiency in the TRD as a function of the electron efficiency. Here, pion efficiency indicates that a pion is misidentified as an electron. The expected TRD performance for a full stack of six layers is shown by the squares and compared to test beam data represented by the circles.

\section{The ALICE Zero Degree Neutron Calorimeter}

The Zero Degree Neutron Calorimeters (ZDC) are placed on both sides of the interaction point at a distance of $116 \mathrm{~m}[3$. The ZDC information can be used to select different diffractive topologies. Events of the type $p p \rightarrow p p X$ do not deposit energy in these calorimeters, events $p p \rightarrow$ $p N^{*} X$ will have energy in one of the calorimeters whereas events $p p \rightarrow N^{*} N^{*} X$ will have energy deposited in both calorimeters. Here, $\mathrm{X}$ denotes a centrally produced diffractive state from which the diffractive L0 trigger is derived as described below.

\section{The ALICE diffractive gap trigger}

Additional detectors for event classification and trigger purposes are located on both sides of the ALICE central barrel. First, an array of scintillator detectors (V0) is placed on both sides of the central barrel. These arrays are labeled V0A and V0C on the two sides, respectively. Each of these arrays covers a pseudorapidity interval of about two units with a fourfold segmentation of half a unit. The azimuthal coverage is divided into eight segments of $45^{0}$ degrees hence each array is composed of 32 individual counters. Second, a Forward Multiplicity Detector (FMD) is located on both sides of the central barrel. The pseudorapidity coverage of this detector is $-3.4<\eta<-1.7$ and $1.7<\eta<5.1$, respectively.

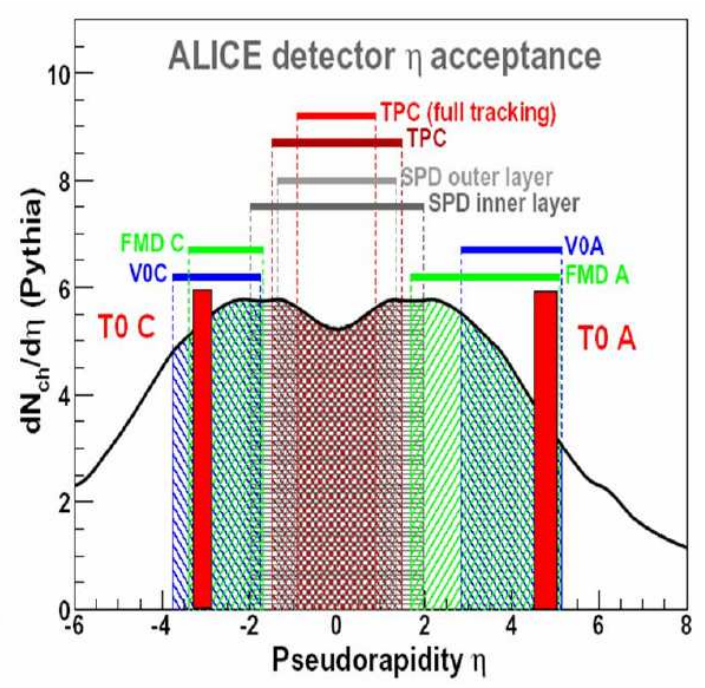

Figure 4. Pseudorapidity coverage of trigger detectors and of detectors in central barrel 
Fig 4 shows the pseudorapidity coverage of the detector systems described above. The geometry of the ALICE central barrel in conjunction with the additional detectors V0 and FMD is well suited for the definition of a rapidity gap trigger. The ALICE trigger system is designed as a multilevel scheme with L0,L1 and L2 levels and a highlevel trigger (HLT). A central trigger processor (CTP) collects the information from the different detector systems and distributes the L0,L1 and L2 decisions back to the detectors. For L0 decision, the trigger information has to arrive at the CTP 850 ns after the interaction time. A rapidity gap trigger can be defined by the requirement of signals coming from the central barrel detectors while V0 and FMD not showing any activity. Such a scheme requires a trigger signal from within the central barrel for L0 decision. The pixel detector of the ITS system is suited for delivering such a signal 4 .

The TRD detector system needs special consideration in the definition of a rapidity gap trigger. The TRD readout electronic is partly put in sleep mode after readout of an event in order to reduce power consumption. The TRD detector hence has its own pretrigger system which generates a wake up signal prior to an L0 decision from CTP. This pretrigger system has access to the information of the $\mathrm{V} 0$ detectors and generates wake up calls based thereupon. The V0 signal is, however, absent in a diffractive trigger. The pixel information described above is late for a diffractive TRD wake up call. Such a diffractive TRD wake up call can be generated based upon the information of the TOF detector. The information of the TOF array is forwarded to the TRD pretrigger system where multiplicity conditions and topological constraints are defined. In addition, the information of the V0 detectors is available at this level hence the rapidity gap width can be defined. The resulting L0 signal is fast enough to reach the CTP well before the time limit for L0 decision.

The high level trigger HLT has access to the information of all the detectors shown in Fig 4 and will hence be able to select events with rapidity gaps in the range $-4<\eta<-1$ and $1<\eta<5$. These gaps extend over seven units of pseudora- pidity and are hence expected to suppress minimum bias inelastic events by many orders of magnitude.

In addition to the scheme described above, the ALICE diffractive L0 trigger signal can be generated from the Neutron ZDC if no central state is present in the reaction. A L0 signal from ZDC does, however, not meet the 850 ns L0 time constraint described above. A L0 trigger from $\mathrm{ZDC}$ is therefore only possible during special data taking runs for which the $850 \mathrm{~ns}$ time limit is extended by about 150 ns. The possibility of such data taking is currently under discussion.

\section{ALICE diffractive and electromagnetic physics}

The tracking capabilities at very low transverse momenta in conjunction with the excellent particle identification make ALICE an unique facility at LHC to pursue a long term physics program of diffractive and electromagnetic physics. The low luminosity of ALICE as compared to the other LHC experiments restricts the ALICE physics program to reactions with cross section at a level of a few nb per unit of rapidity.

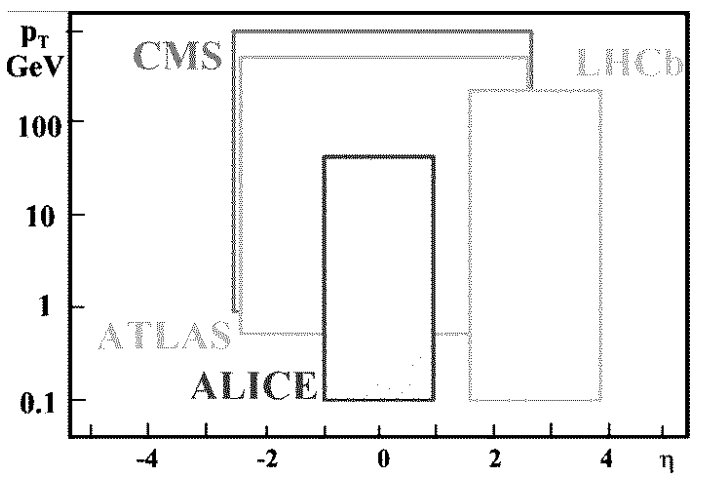

Figure 5. Rapidity and transverse momentum acceptance of the LHC experiments

Fig $[5$ shows the transverse momentum acceptance of the four main LHC experiments. Not 
shown in this figure is the acceptance of the TOTEM experiment which has a physics program of measurements of total cross section, elastic scattering and soft diffraction 5 . The acceptance of the TOTEM telescopes is in the range of $3.1<|\eta|<4.7$ and $5.3<|\eta|<6.5$. The combined data taking of TOTEM and CMS represents the largest rapidity interval covered at the LHC. The CMS transverse momentum acceptance of about $1 \mathrm{GeV} / \mathrm{c}$ shown in Fig 5 represents a nominal value. The CMS analysis framework foresees the reconstruction of a few selected data samples to values as low as $0.2 \mathrm{GeV} / \mathrm{c} 6$.

\section{Signatures of the Odderon}

The Odderon was first postulated in 1973 and is represented by color-singlet exchange with negative C-parity[7]. Due to its negative C-parity, Odderon exchange can lead to differences between particle-particle and particle-antiparticle scattering. In QCD, the Odderon can be a three-gluon object in a symmetric color state. Due to the third gluon involved in the exchange, a suppression by the coupling $\alpha_{s}$ is expected as compared to the two gluon Pomeron exchange. However, finding experimental signatures of the Odderon exchange has so far turned out to be extremely difficult 8 . A continued non-observation of Odderon signatures would put considerable doubt on the formulation of high energy scattering by gluon exchange [9]. The best evidence so far for Odderon exchange was established as a difference between the differential cross sections for elastic $p p$ and $p \bar{p}$ scattering at $\sqrt{s}=53 \mathrm{GeV}$ at the CERN ISR. The $p p$ cross section displays a dip at $\mathrm{t}=-1.3 \mathrm{GeV}^{2}$ whereas the $p \bar{p}$ cross section levels off. Such a behaviour is typical for negative C-exchange and cannot be due to mesonic Reggeons only.

\subsection{Signatures of Odderon Cross Sections}

Signatures of Odderon exchanges can be looked for in exclusive reactions where the Odderon (besides the photon) is the only possible exchange. Diffractively produced C-even states such as pseudoscalar or tensor mesons can result from photon-photon, photon-Odderon and Odderon-Odderon exchange. Any excess mea- sured beyond the well understood photon-photon contribution indicates an Odderon contribution.

Diffractively produced C-odd states such as vector mesons $\phi, J / \psi, \Upsilon$ can result from photonPomeron or Odderon-Pomeron exchange. Any excess beyond the photon contribution would be indication of an Odderon exchange.
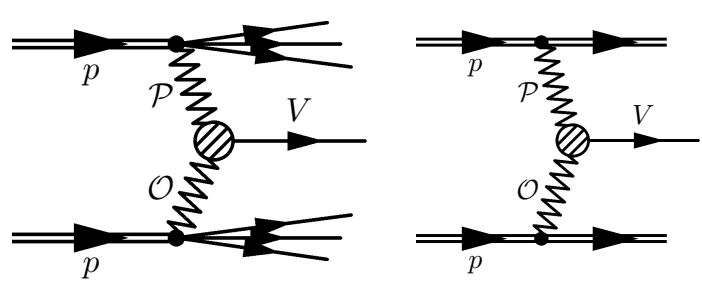

Figure 6. Vector Meson production by OdderonPomeron fusion

Fig 6 shows the Feynman diagram for vector meson production by Pomeron-Odderon fusion with breakup of the protons on the left and without breakup on the right. The two different reaction channels can be identified by the information of the ZDC. To each of the two diagrams in Fig 6 exists a diagram in which the Odderon is replaced by a photon.

Cross sections for diffractively produced $J / \psi$ in pp collisions at LHC energies were first estimated by Schäfer et al[10]. More refined calculations by Bzdak et al result in a t-integrated photon contribution of $\left.\frac{d \sigma}{d y}\right|_{y=0} \sim 15 \mathrm{nb}$ and a t-integrated Odderon contribution of $\left.\frac{d \sigma}{d y}\right|_{y=0} \sim 1 \mathrm{nb}[11$. These two numbers carry large uncertainties, the upper and lower limit of these numbers vary by about an order of magnitude. This cross section is, however, at a level where in $10^{6} \mathrm{~s}$ of data taking in ALICE the $J / \psi$ can be measured in its $\mathrm{e}^{+} \mathrm{e}^{-}$ decay channel at a level of $4 \%$ statistical uncertainty. Due to the different t-dependence, the two contributions result in different $p_{T}$ distributions of the $J / \psi$. The photon and Odderon contributions are shown in Fig.7 by the dotted and solid 
lines, respectively. A careful transverse momentum analysis of the $J / \psi$ might therefore allow to disentangle the Odderon contribution.

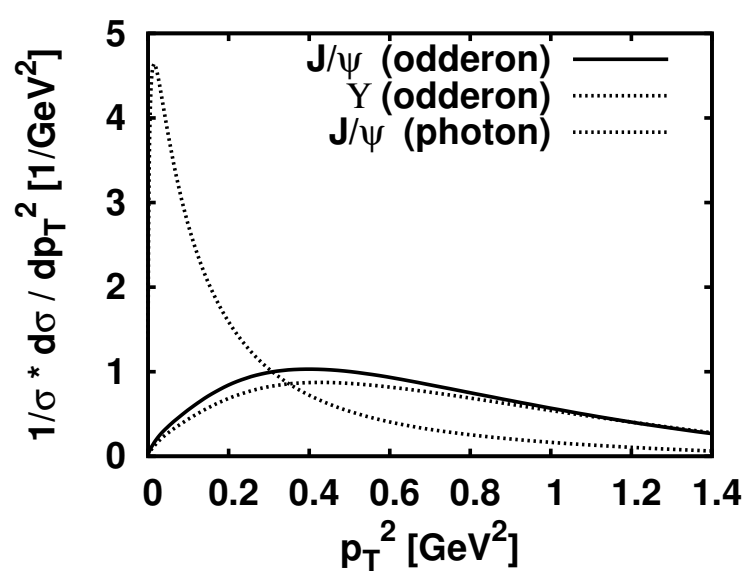

Figure 7 . The $\mathrm{J} / \psi$ transverse momentum distribution for the photon and Odderon contributions

\subsection{Signatures of Odderon Interference Effects}

If the diffractively produced final state is not an eigenstate of C-parity, then interference effects between photon-Pomeron and photon-Odderon amplitudes can be analyzed.

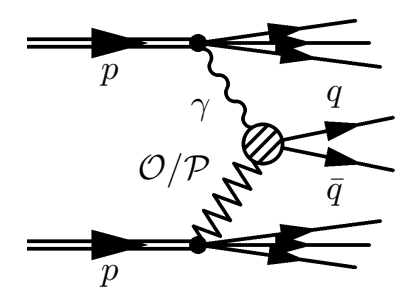

Figure 8. photon-Pomeron and photon-Odderon amplitudes
Fig 8 shows the photon-Pomeron and the photon-Odderon amplitudes for $q \bar{q}$ production.

A study of open charm diffractive photoproduction estimates the asymmetry in fractional energy to be on the order of $15 \%[12$. The forward-backward charge asymmetry in diffractive production of pion pairs is calculated to be on the order of $10 \%$ for pair masses in the range $1 \mathrm{GeV} / \mathrm{c}^{2}<m_{\pi+\pi-}<1.3 \mathrm{GeV} / \mathrm{c}^{2} \mathbf{1 3 1 4}$.

\section{Acknowledgments}

I thank Otto Nachtmann and Carlo Ewerz for illuminating discussions and Leszek Motyka for preparing and communicating Figure 7.

\section{REFERENCES}

1. F. Carminati et al, ALICE Collaboration, 2004, J.Phys. G: Nucl. Part. Phys. 301517

2. B. Alessandro et al, ALICE Collaboration, 2006, J.Phys. G: Nucl. Part. Phys. 321295

3. R. Arnaldi et al, Nucl. Instr. and Meth. A $\mathbf{5 6 4}(2006) 235$

4. The ALICE collaboration, ALICE experiment at the CERN LHC, accepted for publication in JINST

5. K. Eggert, TOTEM a different LHC experiment, CERN colloquium, feb 21,2008

6. D. d'Enterria et al, Addendum CMS technical design report, J. Phys. G34:2307-2455, 2007

7. L. Lukaszuk, B. Nicolescu, Lett. Nuovo Cim. 8 (1973) 406

8. C. Ewerz, Proceedings XII Rencontres de Blois (2005) 377

9. S. Donnachie, G. Dosch, P.V. Landshoff, O. Nachtmann, Pomeron physics and QCD, Cambridge University Press (2002) 297

10. A. Schäfer, L. Mankiewicz, O. Nachtmann, Phys.Lett. B 272 (1991) 419

11. A. Bzdak, L. Motyka, L. Szymanowski, J.R. Cudell, Phys.Rev. D 75 (2007) 094023

12. S.J. Brodsky, J. Rathsman, C. Merino, Phys.Lett. B 461 (1999) 114

13. P. Hägler, B. Pire, L. Szymanowski, O.V. Teryaev, Phys.Lett. B 535 (2002) 117

14. I.F. Ginzburg, I.P. Ivanov, N.N. Nikolaev, Eur.Phys.J. C5 (2003) 02 